\title{
Langmuir Monolayer of Artificial Pulmonary Surfactant Mixtures with an Amphiphilic Peptide at the Air/Water Interface: Comparison of New Preparations with Surfacten (Surfactant TA)
}

\author{
Hiromichi Nakahara, Sannamu Lee, Gohsuke Sugihara, Chien-Hsiang Chang, \\ and Osamu Shibata
}

\section{Supplementary data;}

(a)

\section{Hel 13-5: KLLKLLLKLWLKLLKLLL}

\section{KL4: $\quad$ KLLLLKLLLLKLLLLKLLLLK}

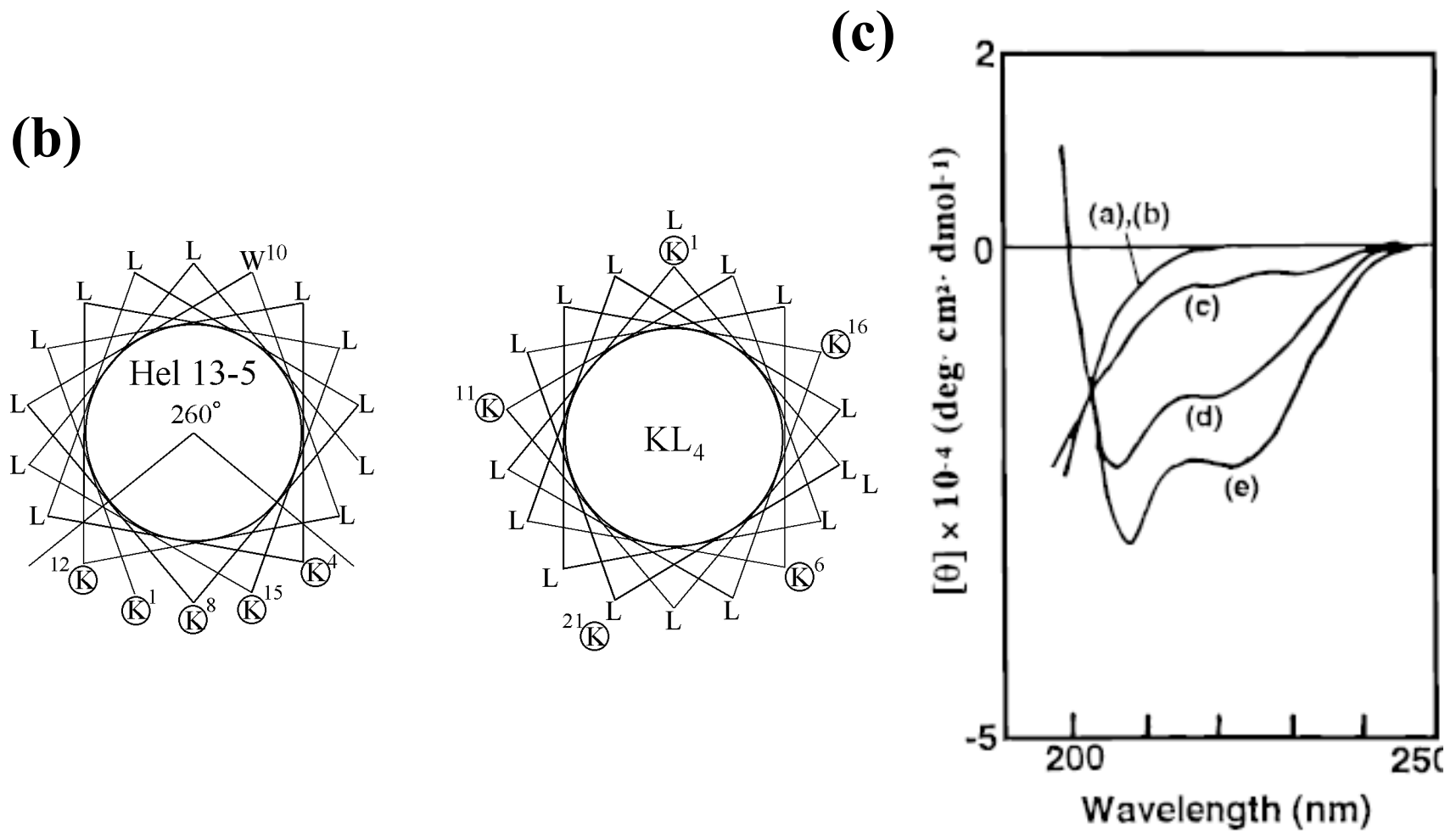

(c)

Figure S1 (a) Primary structures and (b) helical wheel representations of Hel 13-5 and $\mathrm{KL}_{4}$; $\mathrm{L}$ (leucine) and $\mathrm{W}$ (tryptophan) are hydrophobic amino acids, and $\mathrm{K}$ (lysine) is hydrophilic amino acid. The extrapolated area of Hel 13-5 on its $\pi-A$ isotherm was $\sim 2.6 \mathrm{~nm}^{2}$, supporting $\alpha$-helical conformation at the air-liquid interface. More details of orientation of Hel 13-5 during lateral compression at the interface have been reported in the previous paper (Langmuir 2006, 22, 1182-1192). (c) Circular dichroism spectra of amphiphilic model peptides in $5 \mathrm{mM}$ Tris buffer containing $100 \mathrm{mM} \mathrm{NaCl}$ at $\mathrm{pH}$ 7.4. Spectra were measured at $20 \mu \mathrm{M}$ of peptides. Hels 5-13, 7-11, 9-9, 11-7, and 13-5 are symbolized as (a), (b), (c), (d), and (e), respectively (Biochemistry 1996, 35, 13196-13204). 


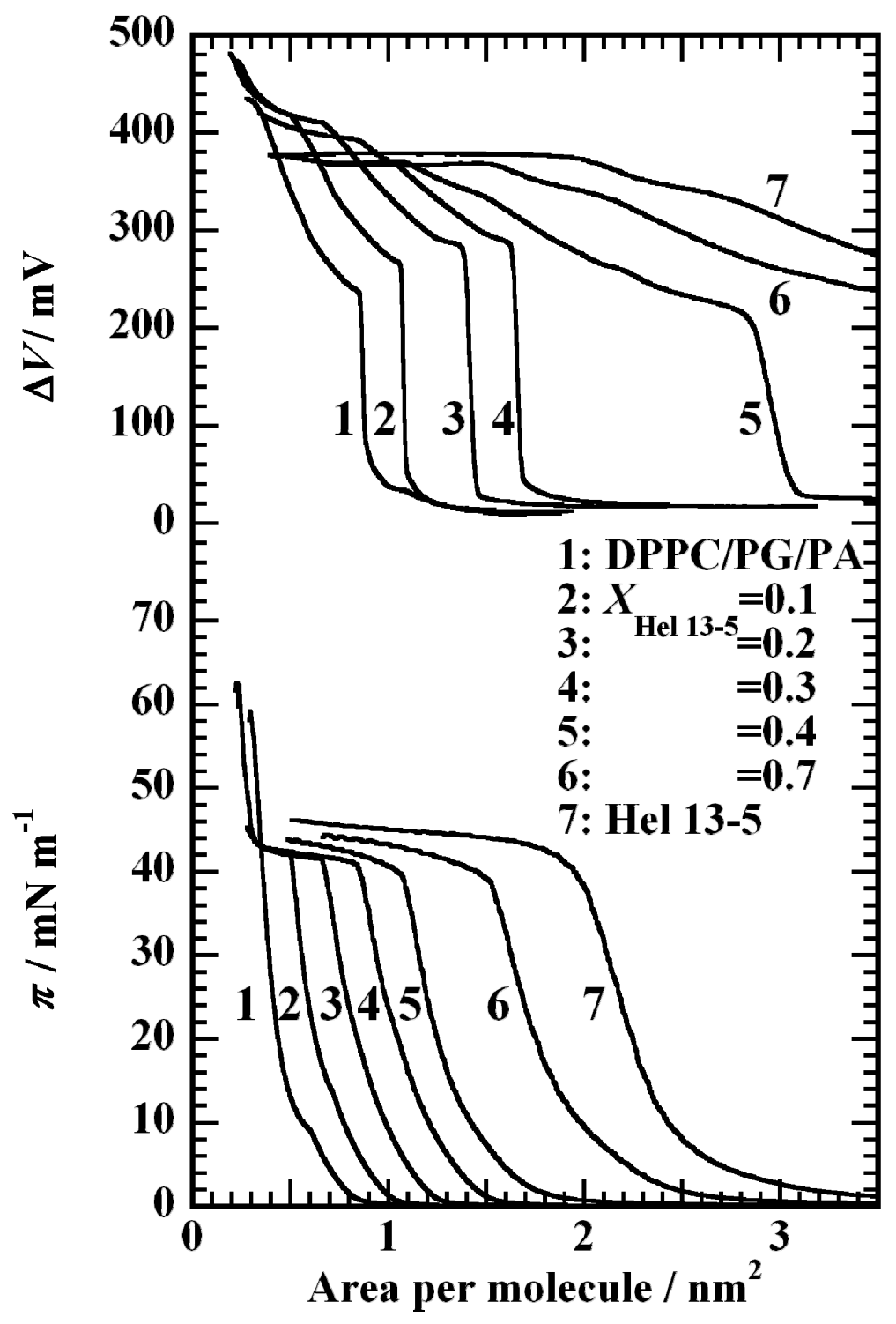

Figure S2

The $\pi$, and $\Delta V-A$ isotherms of the $\mathrm{DPPC} / \mathrm{PG} / \mathrm{PA} / \mathrm{Hel} 13-5$ preparations with the fixed DPPC/PG/PA ratio on a $0.02 \mathrm{M}$ Tris buffer solution (pH 7.4) with $0.13 \mathrm{M} \mathrm{NaCl}$ at $298.2 \mathrm{~K}$. The isotherms are displayed as a function of Hel 13-5. 
A

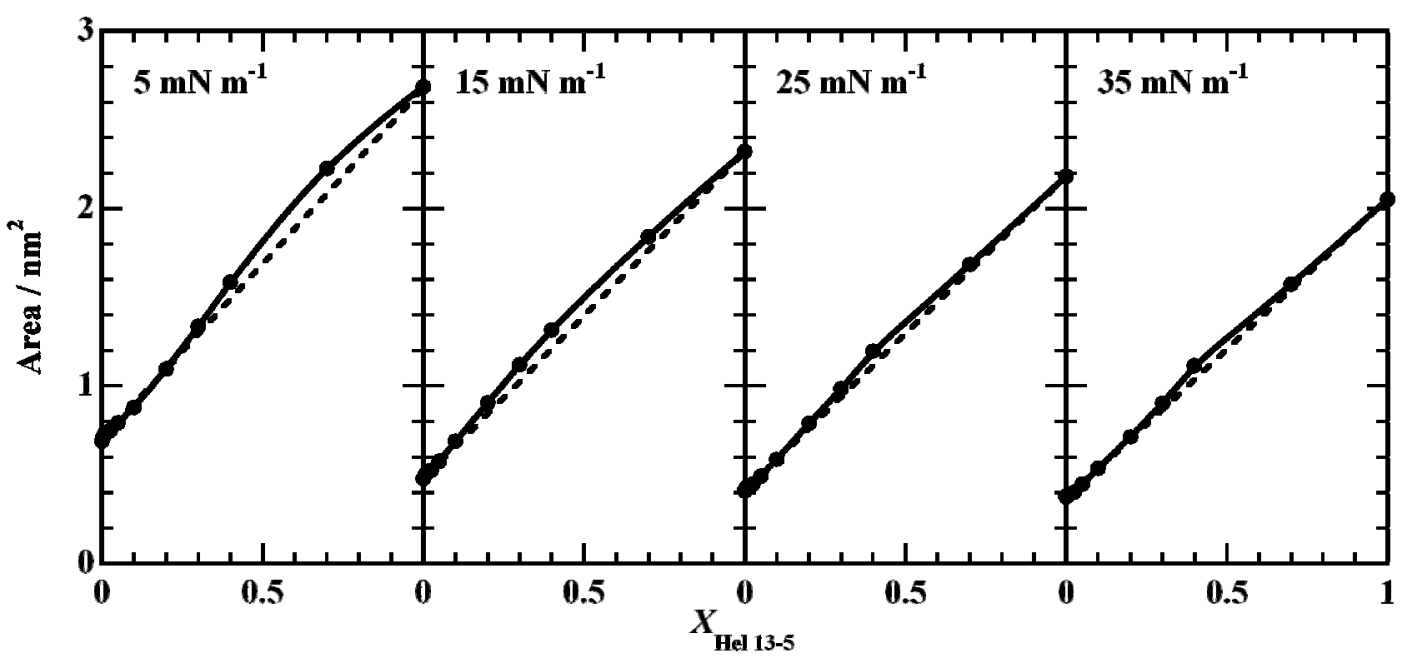

B

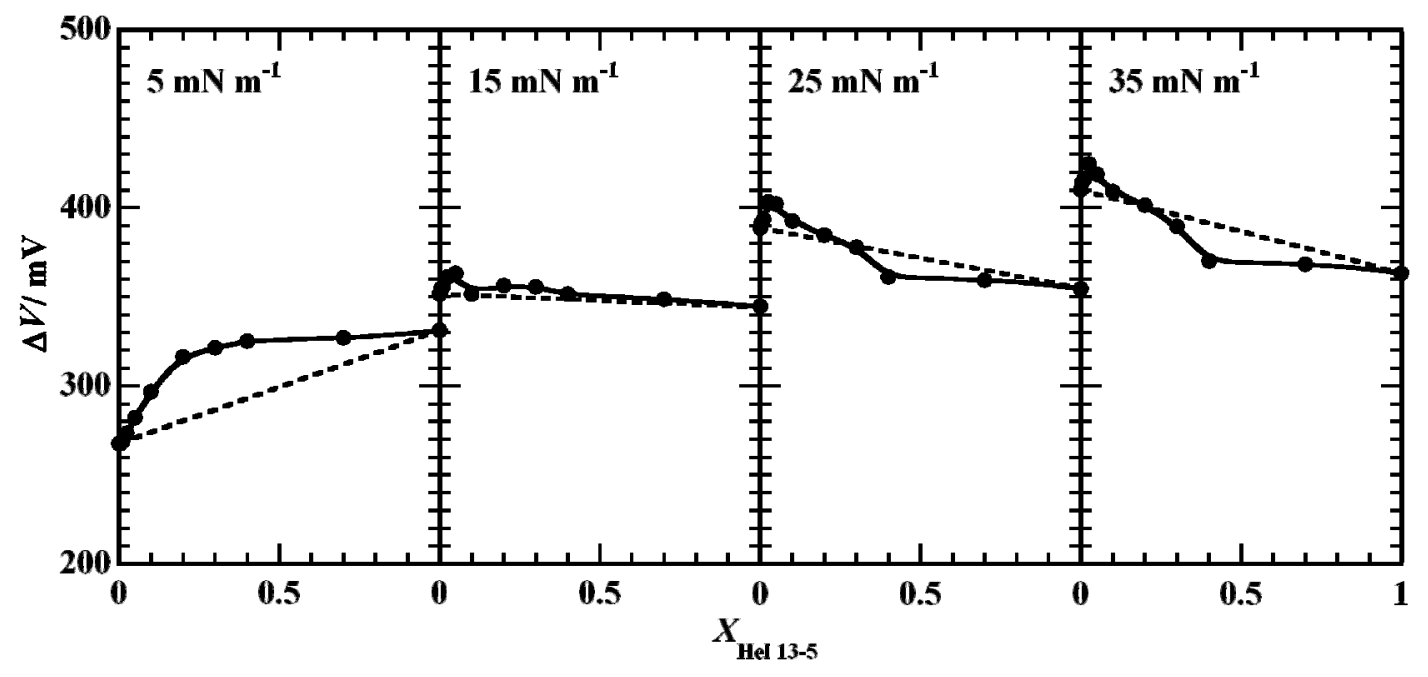

Figure S3 Mean molecular area (A) and Surface potential (B) of the DPPC/PG/PA/Hel 13-5 preparations as a function of $X_{\text {Hel 13-5 }}$ at 5 (a), 15 (b), 25 (c), and $35 \mathrm{mN} \mathrm{m}^{-1}$ (d). The dashed lines were calculated by assuming the additivity rule, and the solid points represent experimental values. 
(a)

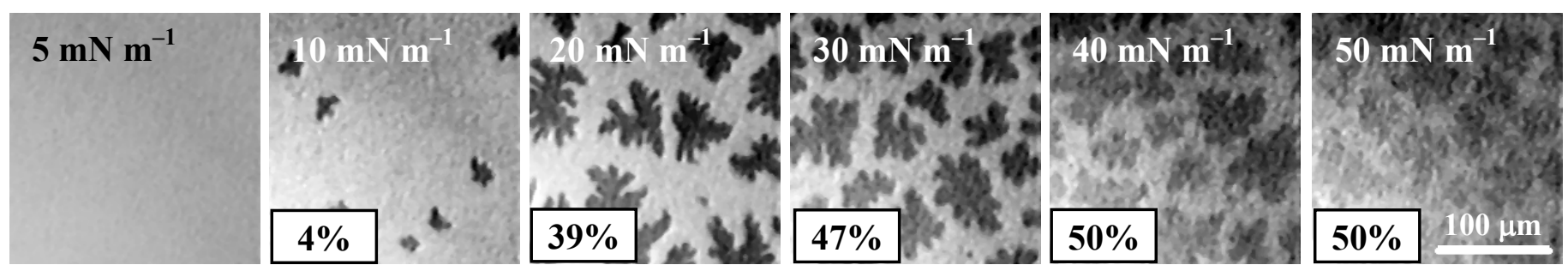

(b)

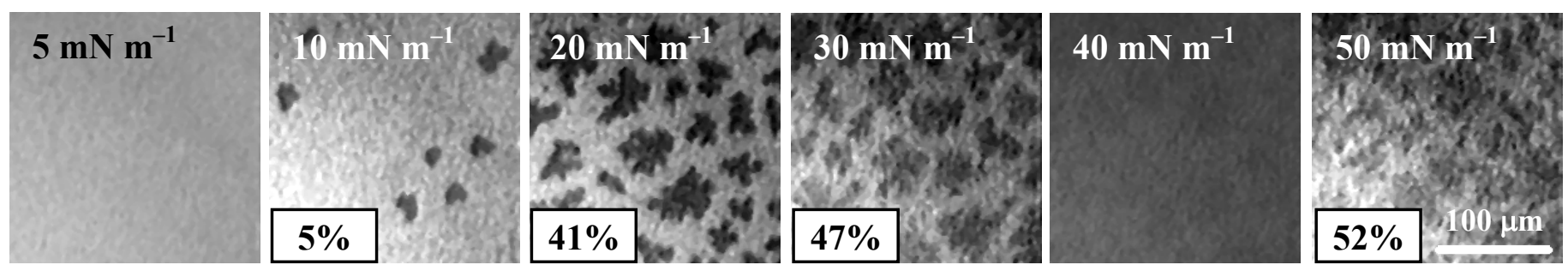

Figure S4 Fluorescent micrographs of the DPPC/PG/PA/Hel 13-5 preparations at $X_{\text {Hel 13-5 }}=0.01$ (a) and 0.025 (b) on a $0.02 \mathrm{M}$ Tris buffer solution ( $\mathrm{pH} 7.4$ ) with $0.13 \mathrm{M} \mathrm{NaCl}$ at $298.2 \mathrm{~K}$. In the coexistence phase, the percentage refers to ordered domains in the micrograph. The monolayers contain $1 \mathrm{~mol} \%$ of fluorescent probe (R18). The scale bar in the lower right represents $100 \mu \mathrm{m}$. 